\title{
Seed Borne Fungal and Bacteria Pathogens Associated with Farmer-Stored Sorghum Seeds from Eastern, Coast and Nyanza Regions in Kenya
}

\author{
Catherine Muui $^{1 *} \quad$ Reuben Muasya ${ }^{2}$ Simon Nguluu ${ }^{2} \quad$ Anne Kambura $^{3} \quad$ Kallen Gacheri $^{1}$ \\ 1. School of Agriculture and Enterprise Development, Kenyatta University, P.O. Box 43844-00100, Nairobi, \\ Kenya \\ 2. School of Agriculture and Veterinary Sciences, South Eastern Kenya University, P.O. Box 170-90200, Kitui, \\ Kenya \\ 3. School of Agriculture, Taita Taveta University, P.O. Box 635 - 80300, Voi, Kenya
}

The research is financed by National Research Fund of Kenya

\section{Abstract}

Sorghum (Sorghum bicolor (L.) Moench) is an important cereal crop in Kenya. Access to good quality seeds is one of the constraints facing the small scale farmers in key areas growing sorghum. Good quality seed is an important factor for maintaining plant population and increasing crop yield to attain food security. One of the important aspects of good seed, besides high germination and purity is the absence of seed borne pathogens. In the present study, an experiment was carried out to identify fungal and bacterial pathogens associated with farmerstored seeds of sorghum. The seeds were collected from farmers in Nyanza, Coast and Eastern regions, Kenya. The seeds were washed with sterile distilled water and sterilized in $1 \%$ Sodium hypochloride for 5 minutes, rinsed with sterile distilled water and dried using a blotting paper. The dried seeds were placed onto prepared Potato Dextrose Agar (PDA) and macerated seeds streaked on nutrient Agar (NA) on sterile Petri dishes and incubated at $23 \pm 2^{\circ} \mathrm{C}$. The growing pathogens were sub-cultured on new media to make pure cultures. A segment of mycelia from the sporulating colonies in each pure culture was examined under a compound microscope and identified based on their morphology, colour and mycelia growth characteristics for fungi while bacterial identification was done using colony color and morphology on nutrient agar and biochemical tests. Ten fungal pathogens and one bacterium were identified growing on the sorghum seed samples. These were Acremonium spp., Alternaria spp., Aspergilus niger, Bipolaris cookie, Collectotrichum graminicola, Fusarium moniliforme, Fusarium solani, Fusarium ventrichosum, Phythium aphanidermatum, Rhizoctonia spp. and a bacterium Xanthomonas spp. It was observed that majority (32\%) of the seed samples from Coast region were infected with bacteria Xanthomonas spp. Phythium. aphanidermatum was the most prevalent fungal pathogen detected on seed samples from Nyanza region with about $20 \%$ prevalence. Results showed that majority of the seed samples from all the regions were contaminated with various pathogens. This study highlights the need to create awareness to farmers on the use of proper production practices, storage facilities and conditions to improve the quality of stored sorghum seeds.

Keywords: farmer-stored seeds, seed-borne pathogens, sorghum

DOI: $10.7176 / \mathrm{JBAH} / 10-18-06$

Publication date:September $30^{\text {th }} 2020$

\section{Introduction}

Sorghum (Sorghum bicolor L) is an important cereal crop cultivated in the semi-arid regions. It is the fifth key cereal crop in the world. Sorghum is mainly cultivated in areas prone to low rainfall and high temperatures making it an important crop in the semi-arid regions such as Eastern, Coast and Nyanza regions in Kenya (Grieser et al., 2006). Being a C4 photosynthetic plant, the fixation of carbon dioxide is effective in sorghum thus making it thrive well in lower latitude zones with higher temperatures and drought (Paterson, 2008). This is a clear indication that sorghum can be used as an alternative crop in areas where other cereal crops such as maize fail in order to achieve food security considering that the crop is used as a staple food for millions of people in the semi-arid tropics (Riziki and Maina, 2013). Similarly, the majority of families who grow sorghum often mill the grain to make ugali among other dishes in order to supplement for maize which is a staple food. Some communities also mix the crop with other grains like cowpeas, amaranthus, and green grams and boil to make traditional meals (Muui et al., 2013; Moses et al., 2016). Sorghum is also used for brewing beer in India and Africa, while in the United States; it is used for production of syrup (Awada, 2016). Furthermore, sorghum can be used as building material, production of brooms, as fodder and animal feed and for production of biofuel (Dahlberg et al., 2012; Rao et al., 2014; Taylor, 2019).

Sorghum production is still low among farmers in Kenya despite the many benefits associated with the crop (Muui et al., 2019). Access to quality planting materials is one of the constraints facing the small scale farmers in the key areas growing sorghum. The quality of seeds is considered as an important factor for increasing yield through higher production per unit area to attain food security of the country (Badigannavar et al., 2016). High 
quality seeds have the ability for efficient utilization of the inputs such as fertilizers and irrigation and eventually maximize yield. In addition, high quality seeds exhibits characteristics such as high germination percentage, free from pests and diseases, high seed vigor, rapid emergence and vigorous growth (Jisha et al., 2013). Seed quality is not a single attribute, but rather comprises a number of discrete components that are critical to growth and productivity of crops (EL-Dahab et al., 2016).

In Kenya, majority of the small scale farmers who venture into sorghum production rely on the farmer saved seeds (Muui, et al., 2019; Kagwiria et al., 2019). This is because of expensiveness of certified seed and can hardly afford to purchase it. Sometimes, the economic gains from using a higher seed quality do not justify the purchase of seed (Almekinders and Louwaars, 2002). However, farmer saved seeds are susceptible to deterioration due to attack by various pathogens during storage conditions (Kagwiria et al., 2019). The seed borne pathogens accumulate in farm saved seeds frequently and may lead to poor germination, poor growth and low yields (Shenge et al., 2007; Syed et al., 2012). Additionally, the seed pathogens also produce mycotoxins that pose health risks to humans and animals (Girish et al., 2004). Therefore, this study was designed to study the prevalence of the seed-borne pathogens associated with farmer saved sorghum seeds collected from the key sorghum growing regions of Eastern, Coast and Nyanza, Kenya.

\section{Materials and Methods}

\subsection{Experimental Location}

Sorghum seed accessions were collected from farms located in Eastern, Nyanza and Coast regions of Kenya in 2018 while conducting a baseline survey (Muui et al., 2019). The distribution of the farms ensured that all the agro ecological zones in each region were represented. Purposive sampling method was used to identify farmers growing sorghum in Nyanza, Eastern and Coast regions, Kenya. A total of 76 farmers were reached from whom 108 farmer-stored sorghum seed accessions were randomly collected comprising of 41 accessions from Eastern, 25 from Nyanza and 42 from Coast (Muui et al., 2019, 2020). A composite sample of each seed accession obtained from the farmers was prepared by mixing the samples and preserving them in Khaki bags at the laboratory temperature for use in the isolation and detection of seed-borne pathogens at Kenyatta University which is situated in Nairobi County about $20 \mathrm{Km}$ from Nairobi city along Nairobi-Thika road between August and October 2018.

\subsection{Isolation and Identification of Sorghum Seed Pathogens}

A sample of 400 seeds was taken from each of the 108 sorghum seed accessions (Table 1). Seeds were arranged in four replicates of 100 seeds. The seeds were surface sterilized using $70 \%$ ethanol by submerging in the solution for $1 \mathrm{~min}$ to eliminate surface contaminants; then dried using blotter papers. Fungal isolation was done by placing the surface sterilized seeds on Potato Dextrose Agar (PDA) media in sterile petri dishes that were sealed using parafilm. The media was prepared by dissolving 39 grams of PDA in $1000 \mathrm{ml}$ of distilled water. The solution was put in an autoclave (UTKBS-V Series) at $121{ }^{\circ} \mathrm{C}$ for 15 minutes at 15 pound pressure, then left to cool to $45^{\circ} \mathrm{C}$ in a water bath to avoid killing the pathogens. The samples were then incubated upside down at a room temperature of $23^{\circ} \mathrm{C}( \pm 2)$ for 7 days (Amusa et al., 2007; Baiyewu et al., 2007). The resulting pathogens were sub-cultured on new PDA media then incubated at a room temperature of $23^{\circ} \mathrm{C}( \pm 2)$ for 7 days (Gwary et al., 2006; Abdulsalaam and Shenge, 2011). The growing fungal colonies were examined to identify the pathogens using their growth habits, colonial morphology, mycelial structure, spores and associated structures according to the keys of Boucias et al, (2007), Roy et al, (2001), Okey (2015). Slide preparations of the various fruiting structures were also made and observed under a compound microscope (XSZ-107T) at a magnification X40 for characteristics of their vegetative and reproductive structures such as hyphae type, colour, size, shape of conidia and conidiophores. The various types of pathogens were identified using identification keys (Watanabe, 2010; Abdulsalaam and Shenge, 2011).

To isolate and identify bacteria, Nutrient agar (NA) was the standard media used to isolate the pathogens from the sorghum seeds. Twenty eight grammes of Nutrient Agar were used to constitute the media. The seed samples were first washed under a running tap water, then dipped into $1 \%$ sodium hypochlorite for three minutes and rinsed in three changes of sterile distilled water. Sterile blotting papers were used to dry the seeds. Using a sterile universal bottle the seeds were macerated using a sterile stirring rod where a sterile loop was used to get some segments of the seed and streaked on the Nutrient Agar (NA) in Petri dishes. Colony formation was observed after 48 hours. Bacterial colonies that appeared were sub-cultured in sterile NA plates to obtain single colony for identification and maintenance. The single purified colonies of bacteria were streaked on NA slants for further confirmation and preservation.

Biochemical tests were done for identification of bacteria where type and activity of enzymes produced by the bacteria characteristics were used for the identification of the microorganisms. The bacteria were first identified using colony color and morphology on nutrient agar (NA) according to Shaker (2014). Gram's staining was performed as first discriminatory test for the subject bacterial pathogen (Moyes et al., 2009). Bacteria was smeared on the sterile microscopic slide and fixed by heating over the flame. Crystal violet and Gram's iodine stains were applied and washed with water after one minute and then acetone added to remove Gram's iodine 
stain. The smear was again washed with water. Safranin stain was later applied and washed with water after one minute, blotted dry and colonies observed under the microscope. Indole Production Test, Methyl red (MR) test, Voges - Proskaure (VP) test and Citrate utilization test were all done for identification and confirmatory of the bacteria.

\subsection{Data Analysis}

The frequencies and percentage of abundance was generated. The percentage frequency (PF) of the pathogens identified was calculated as described by Bisrat et al (2014). $\mathrm{PF}=$ (Number of seeds on which pathogens appear/Total number of seeds cultured) X 100

\section{Results}

\subsection{Seed Borne Pathogens Identified on Farmer-Saved Sorghum Seeds Obtained from Nyanza, Coast and} Eastern Regions of Kenya

The results of the study showed that various pathogens had infected the various sorghum seeds obtained from Nyanza region (Fig. 1 \& 2). The identified pathogens were: Xanthomonas spp, Colletotrichum graminicola, Fusarium moniliforme, Fusarium solani, Aspergillus niger, Alternaria spp, Fusarium ventricusum, Bipolaris cookie, Acremonium spp and phythium aphanidermatum. Xanthomonas spp was the most prevalent pathogen infecting seeds from all the sorghum accessions. Colletotrichum graminicola was the second most prevalent pathogen isolated in fourteen of the sorghum seed accessions. F. moniliforme was the third most prevalent pathogen which was isolated in 8 sorghum accessions. However, Fusarium solani and Acremonium spp infected one accession each meaning they were rare pathogens in Nyanza region. Bipolaris cookie and Phythium aphanidermatum were identified in two of the 25 accessions while Aspergillus niger and Fusarium ventricusum had infected three.

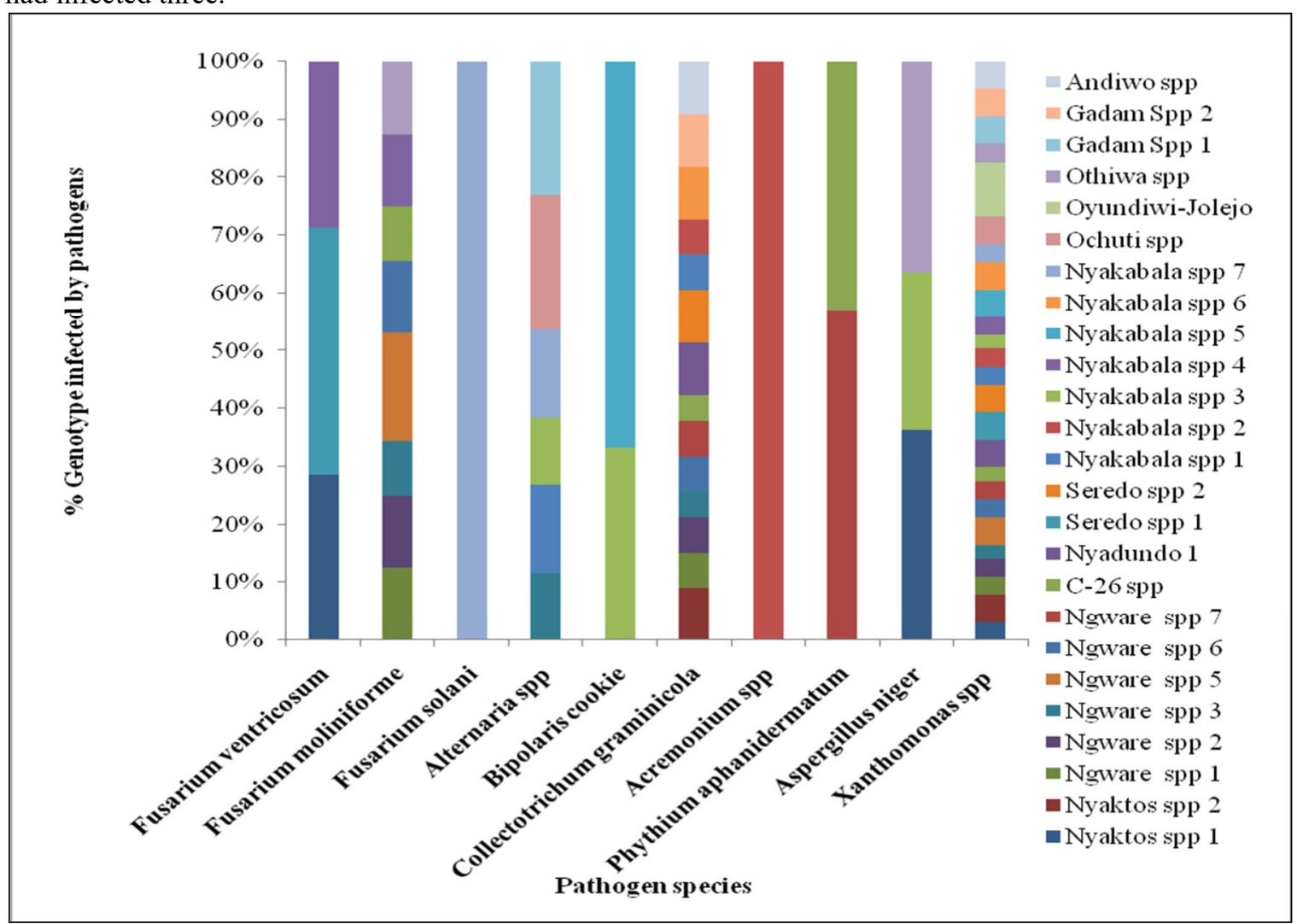

Figure 1: Percentage frequency of genotypes infected by fungi and bacteria in Nyanza region 


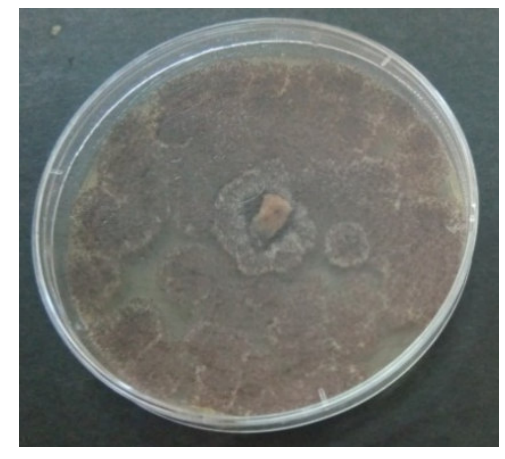

Plate 1: Aspergillusniger

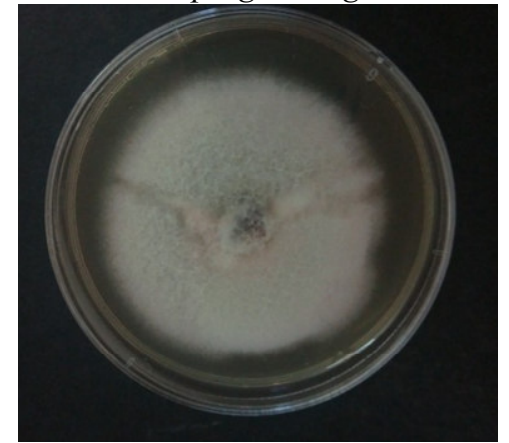

Plate 3: Collectotrichum graminicola

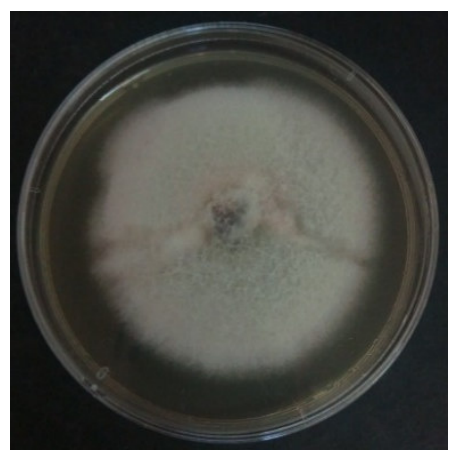

Plate 2: Phythium aphanidermatum.

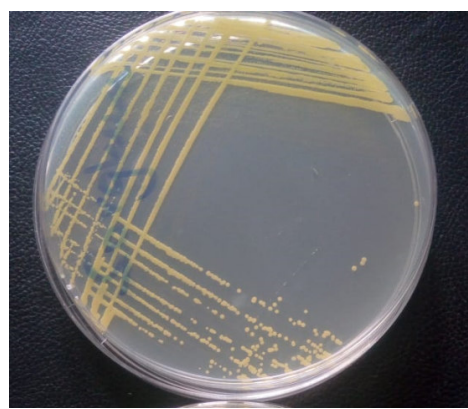

Plate 4: Xanthomonas spp.

Figure 2: Some plates of seed-borne pathogens identified on farmer-stored sorghum seeds

Results obtained from this study showed that seeds of sorghum accessions from coast region were infected by various fungal pathogens and one bacteria (Fig. 3). The pathogens identified in the seeds of the sorghum accessions were: Xanthomonas spp, Colletotrichum graminicola, Fusarium moniliforme, Aspergillus niger, Alternaria spp, Fusarium ventricusum, Bipolaris cookie, Rhizoctonia spp, Acremonium spp and phythium aphanidermatum. Xanthomonas spp, was the most prevalent pathogen that infected the highest number of the genotypes. Alternaria spp was the second most prevalent pathogen that was isolated in eight of the sorghum accessions. Aspergillus niger was isolated in eight of the sorghum seed accessions. Phythium aphanidermatum was isolated from five while Fusarium ventricusum was present in four of the sorghum accessions. Bipolaris cookie and Acremonium spp had infected two sorghum accessions each while Rhizoctonia spp was isolated from only one. 


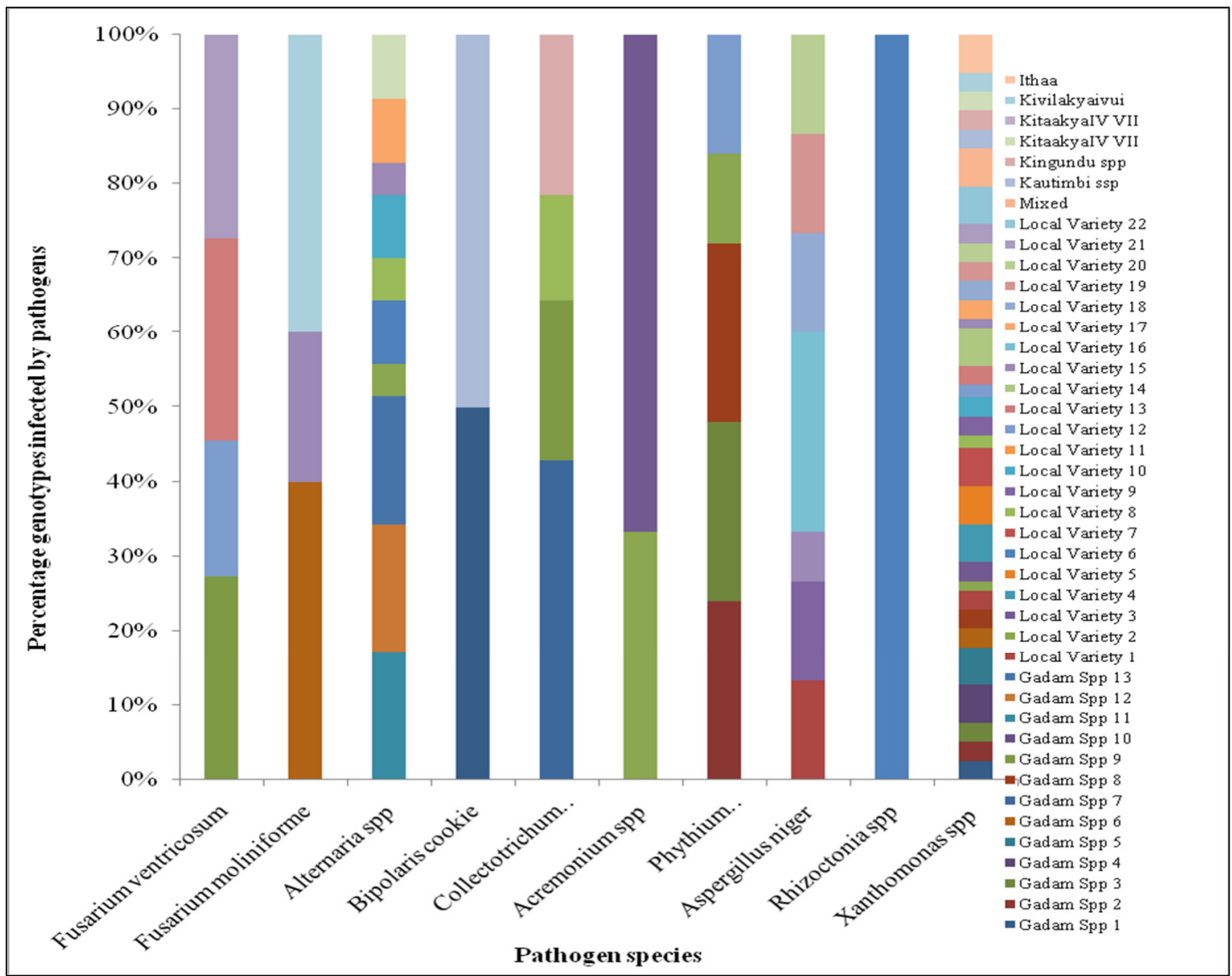

Figure 3: Percentage frequency of genotypes infected by fungi and bacteria in Coastal region

The results indicated that there were pathogens present in all the sorghum seed accessions obtained from eastern region (Fig. 4). The following pathogens were identified from various sorghum seed accessions: Xanthomonas spp, Colletotrichum graminicola, Fusarium moniliforme, Fusarium solani, Aspergillus niger, Alternaria spp, Fusarium ventricusum, Bipolaris cookie and Rhizoctonia spp. Xanthomonas spp was the most prevalent pathogen in the accessions among the pathogens that were identified. Twenty of the sorghum accessions had Xanthomonas spp while Colletotrichum graminicola, was the second most prevalent pathogen isolated from eight accessions. Aspergillus niger appeared in seven of the sorghum genotypes in the Eastern region. Fusarium moniliforme, Alternaria spp and Bipolaris cookie each had infected one sorghum seed accession whereas Fusarium solani and Rhizoctonia spp infected two accessions each. 


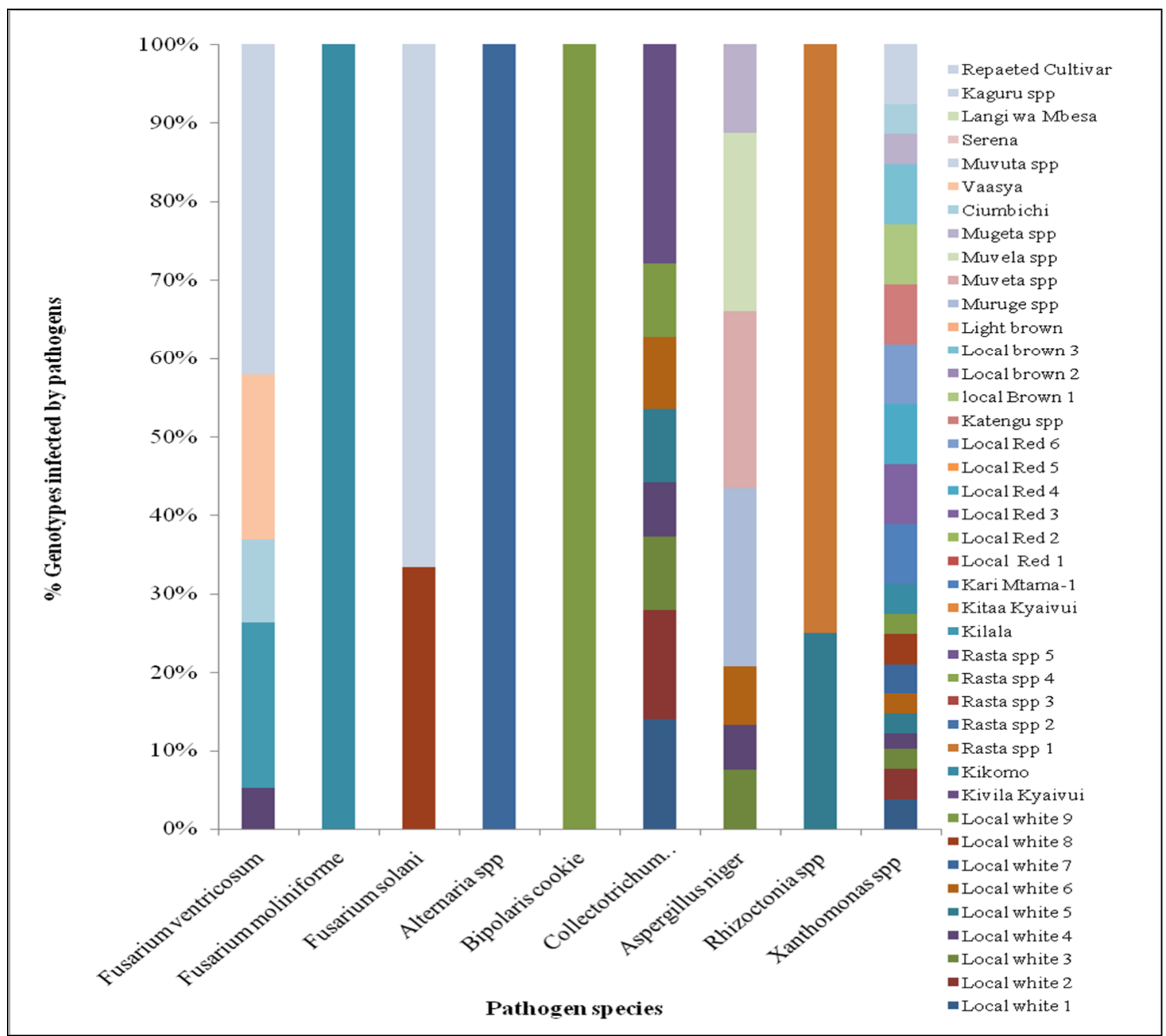

Figure 4: Percentage frequency of genotypes infected by fungi and bacteria in Eastern region

3.2 Percentage Abundance of Fungi and Bacteria Isolated in Nyanza, Coast and Eastern Regions of Kenya The results obtained from this study indicated that Xanthomas spp was the most prevalent pathogen in the three regions with its population being slightly higher in accessions obtained from the Coast region (Fig. 5). Other pathogens varied significantly between the regions. In the Eastern region the second most prevalent pathogen was Aspergillus niger while in the Coast region was Alternaria spp. In the Nyanza region the second most prevalent pathogen was Colletotrichum graminicola. 


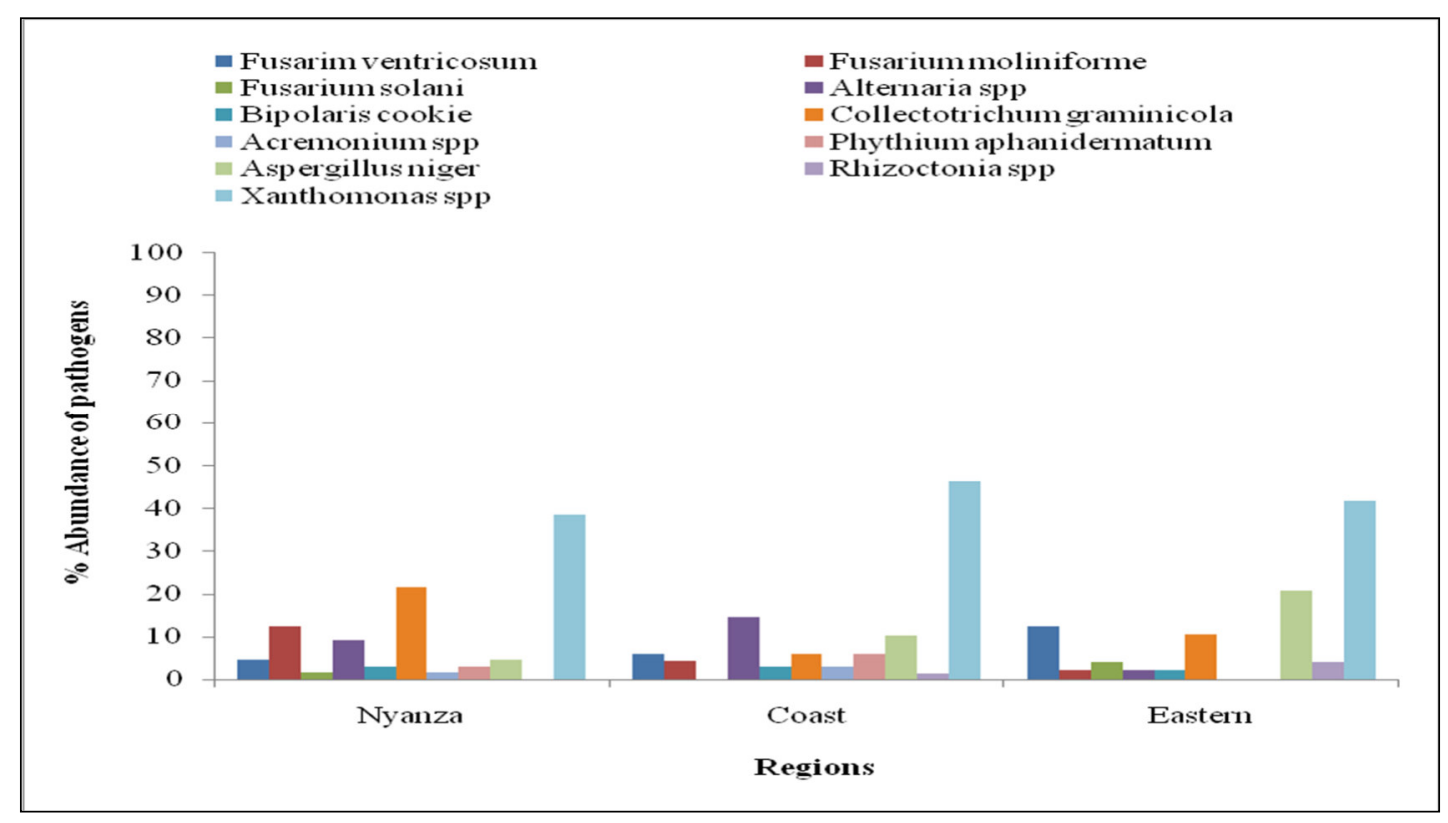

Figure 5: Percentage abundance of fungi and bacteria isolated in Nyanza, Coast and Eastern regions of Kenya

\subsection{Occurrence of Seed-Borne Pathogens on Sorghum Seeds from Nyanza, Coast and Eastern Regions, Kenya}

The results showed that $100 \%$ of the farmer-saved sorghum accessions collected from Nyanza region was infected with at least one pathogen (Table 1). Of the 42 accessions collected from Coast region, about two were free from pathogens accounting for about $23.8 \%$. Results revealed that 23 accessions constituting about $56.1 \%$ obtained from Eastern region were healthy and free from pathogen infections.

\begin{tabular}{|c|c|c|c|c|c|c|c|c|c|c|c|}
\hline Source & Accessions & $\begin{array}{l}\begin{array}{l}\text { Phythium } \\
\text { aphanidermatum }\end{array} \\
\end{array}$ & $\begin{array}{l}\text { Aspergillus } \\
\text { niger }\end{array}$ & $\begin{array}{l}\text { Fusarium } \\
\text { solani }\end{array}$ & $\begin{array}{l}\text { Fusarium } \\
\text { ventrichosum }\end{array}$ & $\begin{array}{l}\text { Fusarium } \\
\text { moniliforme }\end{array}$ & 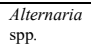 & $\begin{array}{l}\begin{array}{l}\text { Bipolaris } \\
\text { cookie }\end{array} \\
\text { al }\end{array}$ & $\begin{array}{l}\text { Colletotrichum } \\
\text { graminicola }\end{array}$ & $\begin{array}{l}\text { Acremonium } \\
\text { spp }\end{array}$ & $\begin{array}{l}\text { Rhizotonia } \\
\text { spp }\end{array}$ \\
\hline \multirow{25}{*}{ Nyanza } & Nyaktos spp & - & + & - & + & - & - & - & - & - & - \\
\hline & Ngware spp & - & - & - & - & + & - & - & + & - & - \\
\hline & Ngware spp & - & - & - & - & + & + & - & + & - & - \\
\hline & C-26 spp & + & - & - & - & + & - & - & + & - & - \\
\hline & $\begin{array}{l}\text { Nyadundo } 1 \\
\end{array}$ & - & - & - & - & - & - & - & + & - & - \\
\hline & Seredo & - & - & - & + & - & - & - & - & - & - \\
\hline & Nyakabala spp & - & + & - & - & - & + & + & - & - & - \\
\hline & Nyaktos spp & - & - & - & - & - & - & - & + & - & - \\
\hline & Nyakabala spp & - & - & - & + & + & - & - & - & - & - \\
\hline & Ngware spp & - & - & - & - & + & - & - & + & - & - \\
\hline & $\begin{array}{l}\text { Ngware } \\
\text { (white)spp }\end{array}$ & - & - & - & - & + & - & - & - & - & - \\
\hline & Ngware spp & - & - & - & - & + & - & - & + & - & - \\
\hline & Nyakabala spp & - & - & - & - & - & - & + & - & - & - \\
\hline & Nyakabala spp & - & - & - & - & - & - & - & + & - & - \\
\hline & Ochuti spp & - & - & - & - & - & + & - & - & - & - \\
\hline & Nyakabala spp & - & - & - & - & - & + & - & + & - & - \\
\hline & Nyakabala spp & - & - & - & - & - & - & + & - & + & - \\
\hline & $\begin{array}{l}\text { Othiwa spp } \\
\end{array}$ & - & + & - & - & + & - & - & - & - & - \\
\hline & Gadam Spp & - & - & - & - & - & + & - & - & - & - \\
\hline & Nyakabala spp & - & - & + & - & - & + & - & - & - & - \\
\hline & Andiwo spp & - & - & - & - & - & - & - & + & - & - \\
\hline & Ngware spp & + & - & - & - & - & - & - & + & - & - \\
\hline & $\begin{array}{l}\text { Oyundiwi- } \\
\text { Jolejo }\end{array}$ & - & - & - & - & + & - & - & - & - & - \\
\hline & $\begin{array}{l}\text { Seredo spp } \\
\end{array}$ & - & - & - & - & - & - & - & + & - & - \\
\hline & $\begin{array}{l}\text { Gadam Spp } \\
\end{array}$ & - & - & - & - & - & - & - & + & - & - \\
\hline \multirow{26}{*}{ Coast } & Gadam Spp & - & - & - & - & - & - & + & - & - & - \\
\hline & Gadam Spp & - & - & - & - & - & - & - & - & - & - \\
\hline & Local Variety & - & + & - & - & - & - & - & - & - & - \\
\hline & Local Variety & - & - & - & - & - & - & - & - & - & - \\
\hline & Mixed & - & - & - & - & - & - & - & - & - & - \\
\hline & Gadam Spp & + & - & - & - & - & - & - & - & - & - \\
\hline & Gadam Spp & + & + & - & + & - & - & - & - & + & - \\
\hline & Gadam Spp & + & - & - & - & + & - & - & - & - & - \\
\hline & Gadam Spp & - & - & - & - & + & - & - & - & - & - \\
\hline & Local Variety & - & - & - & - & - & - & - & - & + & - \\
\hline & Gadam Spp & - & - & - & - & - & - & - & + & - & - \\
\hline & Kautimbi ssp & - & - & - & - & - & - & + & - & - & - \\
\hline & Gadam Spp & + & - & - & - & - & - & - & - & - & - \\
\hline & Gadam Spp & - & - & - & + & - & - & - & + & - & - \\
\hline & Kingundu spp & - & - & - & - & - & - & - & + & - & - \\
\hline & Local Variety & - & - & - & - & - & - & - & - & - & - \\
\hline & Local Variety & - & - & - & - & - & - & - & - & - & - \\
\hline & Gadam Spp & - & - & - & - & - & - & - & - & - & - \\
\hline & Local Variety & - & - & - & - & - & + & - & - & - & + \\
\hline & Local Variety & - & - & - & - & - & - & - & - & - & - \\
\hline & Gadam Spp & - & - & - & - & - & + & - & - & - & - \\
\hline & $\begin{array}{l}\text { Local Variety } \\
\end{array}$ & - & - & - & - & - & + & - & + & - & - \\
\hline & Local Variety & - & + & - & - & - & - & - & - & - & - \\
\hline & Local Variety & - & - & - & - & - & - & - & - & - & - \\
\hline & Local Variety & - & - & - & - & - & + & - & - & - & + \\
\hline & Kitaakya ivui & - & - & - & - & - & + & - & - & - & - \\
\hline
\end{tabular}




\begin{tabular}{|c|c|c|c|c|c|c|c|c|c|c|c|}
\hline & Gadam Spp & - & - & - & - & - & - & - & - & - & - \\
\hline & Local Variety & + & - & - & + & - & - & - & - & + & 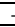 \\
\hline & Local Variety & - & - & - & + & - & - & - & - & - & 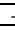 \\
\hline & Kivilakyaivui & - & - & - & - & + & - & - & - & - & - \\
\hline & Local Variety & - & - & - & + & - & - & - & - & - & 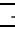 \\
\hline & Local Variety & - & + & - & - & + & + & - & - & - & 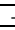 \\
\hline & Local Variety & - & + & - & - & - & - & - & - & - & 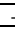 \\
\hline & Local Variety & - & - & - & - & - & + & - & - & 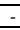 & 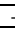 \\
\hline & Gadam Spp & - & - & - & - & - & - & - & - & - & $=$ \\
\hline & Local Variety & - & + & - & - & - & - & - & - & - & 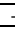 \\
\hline & Local Variety & - & + & - & - & - & - & - & - & - & 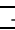 \\
\hline & Local Variety & - & + & - & - & - & - & - & - & - & 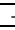 \\
\hline & Local Variety & - & - & - & + & - & - & - & - & + & 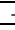 \\
\hline & Local Variety & - & - & - & - & - & - & - & - & - & $=$ \\
\hline & Kitaa Kyaivvii & - & - & - & - & - & + & - & - & - & 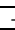 \\
\hline & Ithaa & - & - & - & - & - & - & - & - & - & 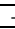 \\
\hline & Local Variety & - & - & - & - & - & - & - & - & - & - \\
\hline Eastern & Local Variety & - & - & - & - & - & - & - & - & - & 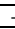 \\
\hline & Local Variety & - & + & - & - & - & - & - & - & - & 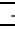 \\
\hline & Kivila Kyaivui & - & + & - & - & - & - & - & - & - & - \\
\hline & Kikomo & - & - & - & - & + & - & - & - & - & $=$ \\
\hline & Rasta & - & + & - & - & + & - & - & - & - & 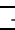 \\
\hline & Kilala & - & - & - & + & - & - & - & - & - & 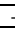 \\
\hline & Local Variety & - & + & - & + & - & - & - & - & - & $=$ \\
\hline & Kitaa Kyaivui & - & - & - & + & - & - & - & - & \pm & 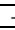 \\
\hline & Kari Mtama1 & - & - & - & - & - & - & - & - & - & 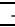 \\
\hline & Local Variety & - & + & - & - & - & - & - & - & - & 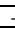 \\
\hline & $\begin{array}{ll}\begin{array}{l}\text { local } \\
\text { Red }\end{array} & \text { Variety } \\
\end{array}$ & - & - & - & - & - & - & - & + & - & 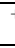 \\
\hline & local variety & - & - & - & - & - & - & - & - & - & - \\
\hline & Katengu & - & - & - & - & - & - & - & - & - & 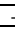 \\
\hline & local Brown & - & - & - & - & - & - & - & - & - & 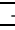 \\
\hline & local Red & - & - & - & - & - & - & - & - & - & 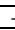 \\
\hline & Rasta spp & - & - & - & - & - & + & - & - & - & $=$ \\
\hline & Muruge spp & - & - & - & - & - & - & - & - & - & 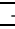 \\
\hline & $\begin{array}{l}\text { Muveta spp } \\
\end{array}$ & - & - & - & - & - & - & - & - & - & 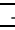 \\
\hline & Muvela spp & - & - & - & - & - & - & - & - & - & 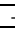 \\
\hline & Mugeta spp & - & - & - & - & - & - & - & - & - & - \\
\hline & Local Red & - & - & - & - & - & - & - & - & - & 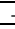 \\
\hline & Local Red & - & - & - & - & - & - & - & - & - & 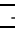 \\
\hline & Ciumbichi & - & - & - & - & - & - & - & - & - & - \\
\hline & $\begin{array}{l}\text { Vaasya } \\
\end{array}$ & - & - & - & - & - & - & - & - & - & $=$ \\
\hline & local brown & - & - & - & - & - & - & - & - & - & 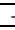 \\
\hline & Rasta & - & - & - & - & - & - & - & - & - & - \\
\hline & Muvuta spp & - & - & - & - & - & - & - & - & - & 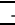 \\
\hline & serena & - & - & - & - & - & - & - & - & - & 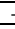 \\
\hline & $\begin{array}{l}\text { Langi wa } \\
\text { Mbesa }\end{array}$ & - & - & - & - & - & - & - & - & - & - \\
\hline & Rasta & - & + & - & - & - & - & - & - & - & 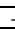 \\
\hline & Kaguru spp & - & - & - & - & - & - & - & - & - & 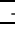 \\
\hline & Rasta spp & - & - & + & - & - & - & - & - & - & 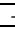 \\
\hline & Light brown & - & - & - & + & - & - & - & - & - & 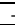 \\
\hline & Local & - & - & - & - & - & + & - & - & - & 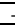 \\
\hline & $\begin{array}{l}\text { Local } \\
\end{array}$ & - & - & + & - & - & - & - & - & - & $=$ \\
\hline & Repeated & - & - & + & - & - & - & - & - & - & 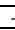 \\
\hline & Local brown & - & - & - & - & - & - & - & - & - & $=$ \\
\hline & $\begin{array}{l}\text { Local Red } \\
\end{array}$ & - & - & - & - & - & - & - & - & - & - \\
\hline & local & - & - & - & - & - & - & + & - & - & 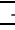 \\
\hline & Local Red & - & - & - & - & - & - & + & - & - & - \\
\hline
\end{tabular}

\section{Discussion}

The findings of this study revealed associations of farmer-stored sorghum seeds with various pathogens across the three regions. All the seed accessions tested were found to be infected with at least one known pathogen. These findings are in agreement with earlier reports by Kamal and Moghal (1968), and Singh, (1983) who reported the presence of Alternaria, Fusarium and Aspergillus species in farm saved sorghum seeds. The results of this study also collaborate with the previous observations of Khan and Bhuta (1994), who reported the prevalence of Fusarium moniliforme as a major pathogen of sorghum seeds. In a related study, Catherine (2014) reported infection of sorghum seeds with various pathogens including Aspergillus niger, Aspergillus flavours and Fusarium spp. Also, similar fungal pathogens were reported on sorghum seeds by Syed et al., (2012) and Owolade et al., (2011).

The widespread contamination of the pathogens in all the sorghum seed accessions collected from Nyanza region compared to the low prevalence of infections on seeds obtained from Coast and Eastern region implied that the seeds could have been exposed to favourable conditions for the development and colonization of the seed pathogens after harvesting (Kange et al., 2014; Kagwiria et al., 2019). The high incidence of fungal seed-borne infections in seeds has also been reported to be associated with higher rainfall and high moisture which creates favourable conditions for fungal growth (Mamiro and Clement, 2012). The low prevalence of seed pathogens on sorghum seeds from Eastern region could be associated with the hot and dry conditions in the region unfavourable for the infestation, colonization and development of the pathogens (Kagwiria et al., 2019).

The high infestation and contamination of the seeds with pathogens during storage have been reported to be responsible for reduction of plant population by about $42 \%$ in the field conditions and hence yield losses (Islam et al., 2009). Fusarium moniliforme for example, reduces crop stands by causing stalk rot, top rot, moldy ears and eventually depreciating yields to a great extent (Osunlaja, 1990). The pathogens also result to the build-up of mycotoxins in infected grains which are harmful to humans and livestock (Fakir, 1999; Abdulsalaam and Shenge, 2011). Most farmers plant sorghum seed which they have retained as grain for consumption on-farm from previous harvests, from neighbours and from local markets (Muui et al., 2013, 2019). Such seed supply system is farmer managed and is based on indigenous knowledge and local diffusion mechanisms. 
Seed health plays an important role in crop establishment and yield of crops. Pathogens associated with seeds not only lower seed viability but also seed vigour resulting in low yield. Earlier studies have reported reduction in yields of upto 15 to $90 \%$ if untreated seeds are grown in the field (Teng et al., 1984). Several pathogens affecting sorghum seeds are known to be responsible for deteriorating seed quality during storage. Populations of most fungi associated with seeds build up in store, therefore basic principles of good handling and storage practices is crucial.

\section{Conclusion}

From the study, Xanthomonas spp., Phythium aphanidermatumand Collectotrichum graminicola were the most prevalent pathogens infecting farmer-stored sorghum seeds with the high prevalence recorded on seeds from Nyanza region followed by Coast and the least infection observed on seeds from Eastern region. The prevalence of various pathogenic fungi and bacteria in farmer-saved seeds of sorghum indicates that there is an urgent need for creation of awareness and capacity building of the small scale farmers on proper post-harvest processing of sorghum seeds, proper storage facilities and conditions to improve the quality of stored seeds. More emphasis should be made on the production, storage and distribution of sorghum to ensure small scale farmers have access to good quality seeds.

\section{Acknowledgements}

The authors acknowledge farmers from Eastern, Nyanza and Coastal regions of Kenya for providing sorghum seed samples and the County agricultural officers for allowing access to the regions. National Research Fund (NRF), Kenya is gratefully acknowledged for funding this study.

\section{References}

Abdulsalaam, S. \& Shenge, K. C. (2011). Seed borne pathogens on farmer saved sorghum (Sorghum bicolor L.) seeds. Journal of Stored Products and Postharvest Research, 2(2), pp. 24 - 28. Available at: http://www.academicjournals.org/JSPPR (Accessed: 2nd September 2020)

Almekinders, C. J. M. \& Louwaars, N. P. (2002). The Importance of the Farmers' Seed systems in a functional seed sector. Journal of New Seeds, 4(1), pp. 15 - 33. Available at: http://dx.doi.org/10.1300/J153v04n01_02 (Accessed: 2nd September 2020)

Amusa, N. A., Kehinde, I. A. \& Ashaya, O. A. (2007). Bio-deterioration of bread fruit (Artocarpus communis) in storage and its effects on the nutrient composition. African Journal of Biotechnology, 1(2), pp. 57-60. Available at: http://www.academicjournals.org/AJB (Accessed: 2nd September 2020)

Awada, F. (2016). Assessment of sorghum response to nitrogen availability. PhD Thesis. University Paris-Saclay. Available at: https://tel.archives-ouvertes.fr/tel-01599245 (Accessed: 2nd September 2020)

Badigannavar, A., Girish, G., Ramachandran, V. \& Ganapathi, T. R. (2016). Genotypic variation for seed protein and mineral content among post-rainy season-grown sorghum genotypes. The Crop Journal, 4(1), 61-67. Available at: https://doi.org/10.1016/j.cj.2015.07.002 (Accessed: 29th August 2020)

Baiyewu, R. A., Amusa, N. A., Ayoola O. A. \& Babalola, O. O. (2007). Survey of the post-harvest diseases and aflatoxin contaminantion of marketed pawpaw fruit (Carica papaya) in south western Nigeria. African Journal of Agricultural Research, 2(4), pp. 178-181. Available at: http://www.academicjournals.org/AJAR (Accessed: 7 th July 2020)

Catherine, M. (2014). Identification and characterization of sorghum (Sorghum bicolor (L.) Moench) landraces and improvement of on-farm seed production in Eastern Kenya. PhD. Thesis. Kenyatta University, Kenya. Available at: http://ir-library.ku.ac.ke/handle/123456789/10967 (Accessed: 7th July 2020)

Dahlberg, J., Berenji, J., Sikora, V. \& Latkovic, D. (2012). Assessing sorghum Sorghum bicolor L. germplasm for new traits: food, fuels and unique uses. Maydica, 56, 2. Available at: https://journalscrea.4science.it/index.php/maydica/article/view/688 (Accessed: 30th August 2020)

Kagwiria, D., Oscar, K. K., Josiah, M. K., George, N. C.\& Henry, F. O. (2019). Sorghum production practices in an integrated crop-livestock production system in Makueni County, Eastern Kenya. Tropical and Subtropical Agroecosystems, 22, pp. 13-23. Available at: https://www.researchgate.net/publication/332871355 (Accesses: 3rd September 2020)

Girish, A. G., Rao, V.P. \& Thakur, R. P. (2004). Diversity of grain mold fungi on selected sorghum genotypes. Indian Phytopathology, 57 (1), pp. 84-87. Available at: http://oar.icrisat.org/id/eprint/4934 (Accessed: 2nd September 2020)

Islam, S. M. M., Masum, M. M. I. \& Fakir, M. G. A. (2009). Prevalence of seed-borne fungi in sorghum of different location of Bangladesh. Scientific Research and Essay, 4(3), pp. 175-179. Available at: http://www.academicjournals.org/SRE (Accessed: 2nd September 2020)

Jisha, K. C., Vijayakumari, K. \& Puthur, J. T. (2013). Seed priming for abiotic stress tolerance: an overview. Acta Physiologiae Plantarum, 35(5), pp. 1381-1396. Available at: https://doi.org/10.1007/s11738-012-1186-5 
(Accessed: 4th September 2020)

Kange, A. M., Erick, K. C., Joshua, O. O., Peter, F. A. \& Sylvans, O. O. (2014). Pre and Post harvest factors affecting sorghum production (Sorghum bicolor L. Moench) among smallholder farming communities. International Journal of Agronomy and Agricultural Research, 5(4), pp. 40-47. Available at: http://www.innspub.net (Accessed:7th July 2020)

Mamiro, D. P. \& Clement, G. (2014). Effect of Sources and Storage Conditions on Quality of Sorghum Seeds. Tanzania Journal of Agricultural Sciences, 13(1), pp. 1-11. Available at: https://www.ajol.info/index.php/tjags/article/view/130228 (Accessed: 3rd September 2020)

Moses, O., James, O., Erick, C., Maurice, O. \& Justice, R. (2016). Utilization of Sorghum Sorghum bicolor L. Moench: A review. International Journal of Agronomy and Agricultural Research, 9(6), pp. 65-76. Available at: http://www.innspub.net (Accessed: 3rd September 2020)

Muui, C. W., Muasya, R. M., Nguluu, S. \& Kambura, A. (2020). Evaluation of seed quality attributes of sorghum germplasm accessions from eastern, coastal and nyanza regions, Kenya. Sustainable Agriculture Research Journal; 9, (3), pp. 9-20. Available at: https://doi.org/10.5539/sar.v9n3p9 (Accessed: 3rd September 2020)

Muui, C. W., Muasya, R. M., Nguluu, S., Kambura, A., Kathuli, P., Mweu, B. \& Odhiambo, D. O. (2019). Sorghum Landraces Production Practices in Nyanza, Coast and Eastern Regions, Kenya. Journal of Economics and Sustainable Development, 10(10), pp. 134-143. Available at: https://www.iiste.org/Journals/index.php/JEDS/article/viewFile/47989/49573 (Accessed: 3rd September 2020)

Muui, C. W., Muasya, R. M. \& Kirubi, D. T. (2013). Baseline survey on factors affecting sorghum production and use in Eastern Kenya. African Journal of Food, Agriculture, Nutrition and Development, 13(1), pp. 73397353. Available at: https://www.ajol.info/index.php/ajfand/article/view/85324 (Accessed: 3rd September 2020)

Owolade, O. F., Olasoji, J. O. \& Afolabi, C. G. (2011). Storage environment and packaging materials on affect seed germination and seed-borne fungi of sorghum (Sorghumbicolor (L.) Moench.) in south west Nigeria. Journal of Environmental, Agricultural and Food Chemistry, 10 (10), pp. 2986-2992. Available at: http://minerva.uvigo.es/.../113 (Accessed: 7th July 2020)

Paterson, A. H. (2008). Genomics of sorghum. International Journal of Plant Genomics. Available at: https://www.ncbi.nlm.nih.gov/pmc/articles/PMC2375965/ (Accessed: 3rd September 2020)

Riziki, M. \& Maina, M. (2013). The potential role of sorghum in enhancing food security in semi-arid Eastern Kenya: A review. Journal of Applied Biosciences, 71, pp. 5786-5799. Available at: https://www.ajol.info/index.php/jab/article/view/98826/88084 (Accessed: 3rd September 2020)

Syed, D. Y. N, Mengesteab, T., Robiel, N., Robiel, W. \& Tecle, Z. (2012). Efficacy of garlic extract and mancozeb against seedborne fungal pathogen of farmer saved sorghum (Sorghum bicolor) and groundnut (Arachis hypogaea) seeds. Journal of Agricultural Sciences 2(2), pp. 31-36. Available at: https://ojs.gjournals.org/index.php/gjas/article/view/14 (Accessed: 3rd September 2020)

Watanabe, T. (2010). Pictorial atlas of soil and seed fungi: morphologies of cultured fungi and key to species. Third edition. Taylor and Francis Group, LLC. Available at: https://www.routledgehandbooks.com/doi/10.1201/EBK1439804193-c3 (Accessed: 7th July 2020) 